\title{
Risk of True Allergy to Local Anesthetics: I0-Year Experience from an Anesthesia Allergy Clinic in China
}

This article was published in the following Dove Press journal: Therapeutics and Clinical Risk Management

\author{
Jun Zuo (D) ${ }^{1,2}$ \\ Ruisong Gong ${ }^{1,2}$ \\ Xiaowen Liu $^{2}$ \\ Jing Zhao ${ }^{1,2}$
}

'Graduate School of Peking Union Medical College, Chinese Academy of Medical Sciences \& Peking Union Medical College, Beijing 100730, People's Republic of China; ${ }^{2}$ Department of Anesthesiology, China-Japan Friendship Hospital, Beijing 100029, People's Republic of China
Correspondence: Jing Zhao

Department of Anesthesiology, ChinaJapan Friendship Hospital, No. 2 Yinghua East Road, Beijing I00029, People's Republic of China

Email zhaojing1009@aliyun.com
Background: Local anesthetics (LAs) have been widely used throughout the healthcare settings, especially in local anesthesia and pain management. The incidence of allergic reactions to LAs remains uncertain. The danger of allergic reactions to the use of LAs in every day of clinical practice is a matter of great concern. Therefore, it is necessary to investigate the risk of true allergy to LAs.

Methods: This study retrospectively evaluated the medical records of patients who were referred to an anesthesia allergy clinic in China and underwent allergy tests with LAs over a 10-year period from 2009 to 2019. The following information was collected from medical records: demographics of the patients, reasons for referral, clinical features of drug hypersensitivity reaction (DHR), and test results with LAs. Skin tests combined with an in vitro method, basophil activation test (BAT), were used to investigate allergic reactions to LAs.

Results: A group of 109 patients were included in the analysis. The main reason for referral was the presence of a suspected DHR after procedures with LAs $(n=68,62 \%)$, the second most common reason for referral was a history of DHR to other drugs and the need to use LAs for upcoming procedures ( $\mathrm{n}=41,38 \%$ ). Of the 68 patients with a suspected DHR to LAs, only six cases presented true allergy and showed positive results in skin tests and/or BAT. And all 41 patients who had a history of DHR to other drugs presented negative in all tests. Conclusion: Risk of true allergy to LAs may be very low. However, patients with a suspected history of DHR to LAs should be considered for allergy tests. Skin tests and BAT may be useful in the investigation and diagnosis of true allergy to LAs in clinical practice.

Keywords: adverse drug reactions, local anesthetics, allergy, skin tests, BAT

\section{Introduction}

LAs are commonly used drugs in every day of clinical practice, including dentistry, delivery, and surgery under local anesthesia, which could make the operation safe and painless. It has shown that adverse drug reactions associated with LAs were usually attributed to vasovagal syncope, overdose toxicity, or a reaction to adrenaline. ${ }^{1}$ However, the indistinct clinical symptoms after administering LAs are often miscalled as "allergic" by doctors, even if there is no evidence in detailed diagnostic tests. ${ }^{2}$ Patients also usually interpret the side-effects as allergy to LAs and have anxiety about the risk of allergic reactions.

The incidence of true allergy to LAs has not been clearly defined. Despite the frequent use of LAs, IgE-mediated allergic reactions have so far been published only in a few convincing case reports. ${ }^{3-5}$ The incidence of true allergy to LAs is 
considered to be rare by allergists, estimated to be about $1 \%{ }^{6-8}$ A recent study pointed out that the risk of allergic reactions to LAs was commonly overestimated. ${ }^{1}$ A Danish national survey over a 10 -year period found that none of 162 patients with suspected perioperative hypersensitivity exposed to LAs had been diagnosed with true allergy. ${ }^{9}$

Allergic reactions to LAs during anesthesia and surgery, especially anaphylaxis, can be life-threatening. It is a challenge for doctors to provide appropriate pain management for patients with true allergy to LAs. Inadequate investigation of suspected allergic reactions to LAs may cause anxiety and discomfort to the patient, as well as ongoing concerns of surgeons and anesthesiologist, which may lead to unnecessary delay in surgery or operation without anesthesia, increasing the risk of procedures for patients. Thus, the study aimed to investigate the risk of true allergy to LAs in our anesthesia allergy clinic over a 10-year period from 2009 to 2019 .

\section{Materials and Methods}

This study retrospectively evaluated all patients who had allergy tests with LAs for any reason over the past 10 years. Cases with incomplete medical records were excluded from the analysis. The study was approved by the Ethics Committee of China-Japan Friendship Hospital. The following information was collected from patients' medical records for each case: demographics of the patients, reasons for referral, the presence of any other allergic diseases, clinical symptoms of drug hypersensitivity reaction (DHR), the history of DHR to LAs and/or other drugs, and test results of suspected and/or requested drugs. The clinical symptoms occurring may involve circulatory symptoms (palpitations, tachycardia, hypotension, shock or cardiac arrest), respiratory symptoms (chest tightness, dyspnea, wheezing, bronchospasm or increased ventilator pressures in intubated patient), cutaneous signs (rash, flushing, erythema, urticaria, or angioedema), and CNS symptoms (anxiety, malaise, sweating, dizziness, somnolence, syncope, or loss of consciousness).

The diagnostic approach was based on the guidelines recommended by the ENDA/EAACI Drug Allergy Interest Group. ${ }^{10-13}$ The diagnostic tests for the allergic reactions to LAs in our anesthesia allergy clinic were performed as follows: skin tests including skin prick test (SPT) and/or intradermal test (IDT), and an in vitro method named basophil activation test (BAT). The patients were advised not to take systemically antiallergenic drugs such as antihistamines, antileukotrienes, and steroids for at least 1 week before all the tests mentioned above. Any drug that induced a positive test result (positive skin tests or BAT) is referred to as the "culprit drug".

\section{Skin Tests}

LAs without vasoconstrictors were used in the skin tests to avoid the appearance of false-positive results. ${ }^{14}$ Drugs tested in this study included procaine, lidocaine, bupivacaine, ropivacaine, and articaine. Maximum concentrations of all drugs in the skin tests are shown in Table 1. All tests were performed with positive histamine control $(10 \mathrm{mg} / \mathrm{mL})$ and negative saline controls $(0.9 \%)$. The positive result of the skin tests, suggestive of an IgE-mediated cause, was defined if the diameter of the wheal was at least $3 \mathrm{~mm}$ larger than the negative control for SPT, and at least $5 \mathrm{~mm}$ larger for IDT. The IDT was performed when SPT was negative.

\section{Basophil Activation Test (BAT)}

In all cases of study, patients' blood was collected into K-EDTA tubes. The blood samples were stimulated with the suspected causative drugs within 4 hours. Concentrations used in BAT are listed in Table 1. Experiments were carried out using the Flow CAST kit (Bühlmann Laboratories AG, Switzerland) following the procedures as previously described. ${ }^{15}$ The samples were analyzed by flow cytometer. Results were considered positive if activated basophil percentage $\geq 5 \%$ and stimulation index (SI=percentage of basophils activated divided by the negative control) $\geq 2$.

\section{Statistics}

The statistical analysis was carried out in software SPSS 23.0 and descriptive data was performed in Microsoft Excel 2016.

\section{Results}

\section{Demographics and Overview of Test}

\section{Results}

A total of 115 patients who were referred to our anesthesia allergy clinic were evaluated in this study, and 109 patients

Table I Maximum Concentrations of LAs in the Skin Tests and BAT

\begin{tabular}{|l|l|l|l|l|}
\hline LAs & $\begin{array}{l}\text { Original } \\
(\mathbf{m g} / \mathbf{m L})\end{array}$ & $\begin{array}{l}\text { SPT } \\
\mathbf{( m g / m L})\end{array}$ & $\begin{array}{l}\text { IDT } \\
\mathbf{( m g / m L})\end{array}$ & $\begin{array}{l}\text { BAT } \\
\mathbf{( m g / m L})\end{array}$ \\
\hline Procaine & 20 & 20 & 2 & $\mathrm{I}$ \\
Lidocaine & 10 & 10 & $\mathrm{I}$ & 0.125 \\
Bupivacaine & 2.5 & 2.5 & 0.25 & 0.5 \\
Ropivacaine & 2 & 2 & 0.2 & $\mathrm{I}$ \\
Articaine & 40 & 40 & 4 & $\mathrm{I}$ \\
\hline
\end{tabular}


( 85 women $/ 24$ men; mean age $=42$ years; range $=6-78$ years $)$ were included in the analysis. However, six patients did not have complete medical records and were excluded (Figure 1).

Most of the 109 patients were female ( $n=85,78 \%$ ). Fortyeight (44\%) out of 109 patients had a history of any other allergic diseases, such as atopy and asthma (Table 2). The main reason for referral was the presence of a suspected DHR after procedures with LAs $(n=68,62 \%)$, the second most common reason for referral was a history of DHR to other drugs and the need to use LAs for upcoming procedures $(n=41,38 \%$ ) (Table 2 and Figure 1). The demographic characteristics of these two group patients are also displayed in Table 2 .

Considering the testing procedures, 99 (91\%) out of 109 patients had skin tests, and $103(94 \%)$ of these patients underwent BAT diagnosis (Table 2). In our clinical practice, all patients with a suspected history of DHR to LAs were tested with the suspected LAs, and all patients who had a history of DHR to other drugs were tested with the requested LAs (Tables 3 and 4). Only six out of these 68 patients with a suspected DHR after procedures with LAs presented true allergy to LAs (two patients had positive skin tests - positive BAT; three patients had positive skin tests - negative BAT; one patient had positive BAT but skin tests were not done) (Figure 1 and Table 5). Meanwhile 62 out of these 68 patients presented negative in all tests. Furthermore, all 41 patients who had a history of DHR to other drugs showed negative results for skin tests and BAT (Figure 1).

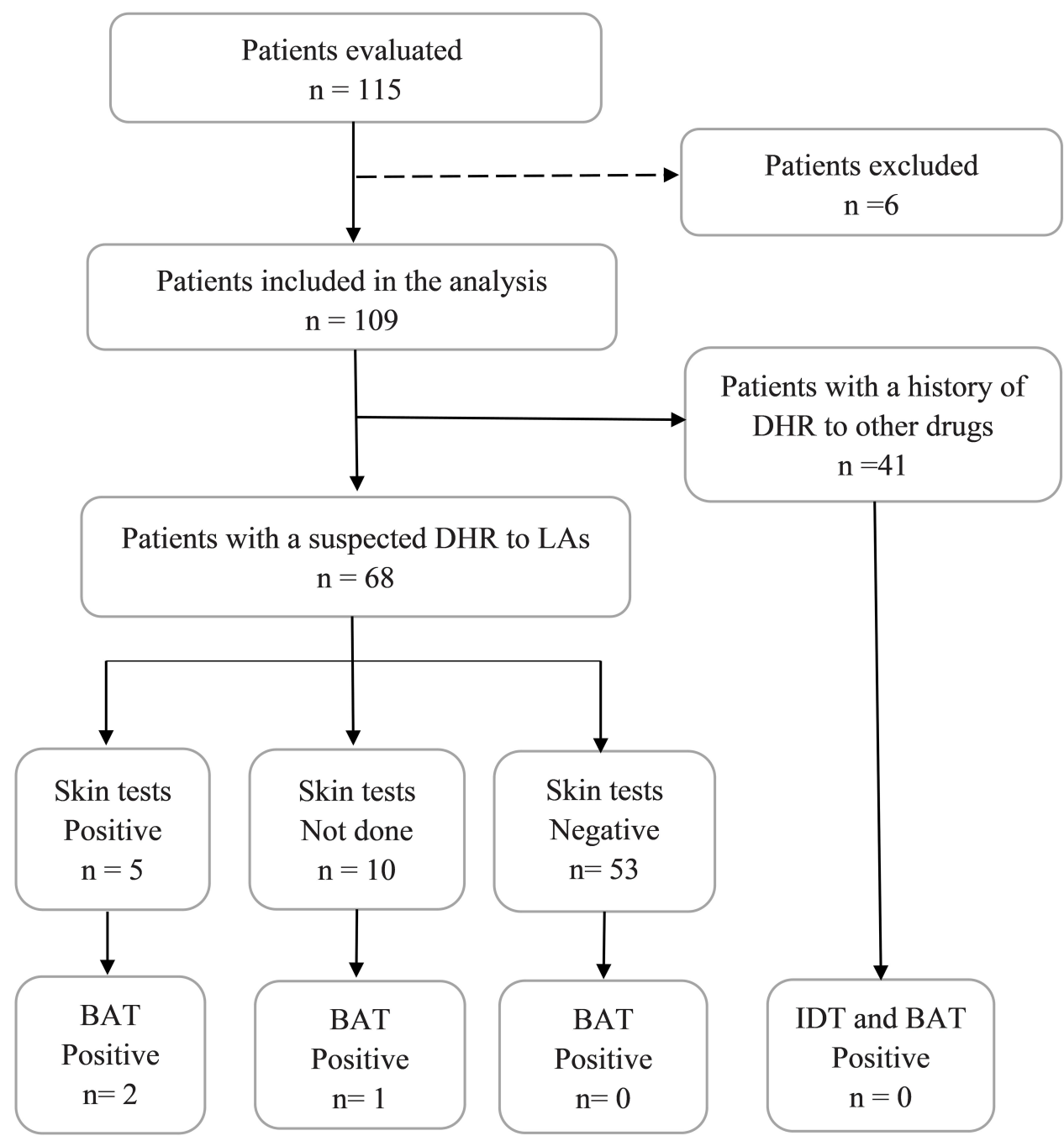

Figure I Flowchart of patients evaluated for allergy to LAs. Abbreviation: $n$, number of patients. 
Table 2 Demographics of the Population Included in the Analysis

\begin{tabular}{|l|l|l|l|}
\hline Variables & Total, $\mathbf{n}(\%)$ & Suspected DHR to LAs & History of DHR to Other Drugs \\
\hline Study population & 109 & $68(62 \%)$ & $41(38 \%)$ \\
Female/male & $85(78 \%) / 24(22 \%)$ & $59(87 \%) / 9(13 \%)$ & $26(63 \%) / 15(37 \%)$ \\
Age, mean (range) & $42(6-78)$ & $43(22-78)$ & $40(6-77)$ \\
History of any other allergic diseases & $48(44 \%)$ & $32(47 \%)$ & $16(39 \%)$ \\
\hline The testing procedures & & & \\
$\quad$ Skin tests & $99(91 \%)$ & $58(85 \%)$ & $41(100 \%)$ \\
BAT & $103(94 \%)$ & $62(91 \%)$ & $41(100 \%)$ \\
\hline
\end{tabular}

\section{Clinical Characteristics of the Study Population}

In this study, 68 patients were exposed to one or more LAs and had a suspected history of DHR to LAs. In most cases,

Table 3 Clinical Characteristics of 68 Patients with a Suspected DHR to LAs

\begin{tabular}{|l|l|}
\hline Variables & n (\%) \\
\hline Suspected LAs & 68 \\
Procaine & $4(6 \%)$ \\
Articaine & $5(7 \%)$ \\
Lidocaine & $31(46 \%)$ \\
Ropivacaine & $2(3 \%)$ \\
Bupivacaine & $1(1 \%)$ \\
Unknown & $25(37 \%)$ \\
\hline Symptoms of drug reactions & \\
Cutaneous & $22(32 \%)$ \\
Rash & $15(22 \%)$ \\
Flushing, erythema & $4(6 \%)$ \\
Urticaria or angioedema & $3(4 \%)$ \\
CNS & $21(31 \%)$ \\
Anxiety, malaise, sweating & $11(16 \%)$ \\
Dizziness & $4(6 \%)$ \\
Somnolence, syncope or loss of consciousness & $8(12 \%)$ \\
Circulatory & $31(46 \%)$ \\
Palpitations, tachycardia & $15(22 \%)$ \\
Hypotension & $7(10 \%)$ \\
Shock or cardiac arrest & $13(20 \%)$ \\
Respiratory & $19(28 \%)$ \\
Chest tightness, dyspnea, wheezing & $16(24 \%)$ \\
Bronchospasm & $3(4 \%)$ \\
Others & $8(12 \%)$ \\
\hline Treatment & $38(56 \%)$ \\
Adrenaline & $25(37 \%)$ \\
Others & $5(7 \%)$ \\
No specified treatment & $15(22 \%)$ \\
\hline Postponed for another procedures & $40(59 \%)$ \\
Unknown & $13(19 \%)$ \\
\hline
\end{tabular}

the suspected drug was lidocaine $(n=31,46 \%)$, whereas the suspected drug was not known in the 25 cases $(37 \%)$ (Table 3). The most common clinical symptoms were circulatory ( $\mathrm{n}=31,46 \%)$, followed by cutaneous symptoms $(n=22,32 \%)$ and CNS symptoms $(n=21,31 \%)$. The exact clinical manifestations of these patients are also shown in Table 3. And 15 (22\%) out of 68 patients who showed allergic reactions to LAs were given to treatment with the intravenous administration of adrenaline. In $38(56 \%)$ cases, the planned procedures were completed, and postponed for another session in 25 (37\%) cases (Table 3).

Considering the 41 patients who had a previous history of DHR to other drugs, the most common drugs for DHR were general anesthetics $(n=25,61 \%)$. In most cases, the requested LAs for upcoming procedures were lidocaine $(n=22,54 \%)$. And lidocaine was tested if requested LAs were unknown by their doctors $(n=15,36 \%)$ (Table 4$)$.

\section{Clinical Data of Six Patients with Test Positivity to LAs}

This study showed that six cases with test positivity to LAs were all women. Four patients had a history of skin reactions after injection of LAs, which were accompanied by respiratory and/or circulatory

Table 4 Overview of 4 I Patients with a History of DHR to Other Drugs

\begin{tabular}{|l|l|}
\hline Variables & $\mathbf{n}(\%)$ \\
\hline History of DHR to other drugs & 41 \\
General anesthetics & $25(61 \%)$ \\
Antibiotics & $9(22 \%)$ \\
NSAIDs & $2(5 \%)$ \\
Radiocontrast agent & $1(2 \%)$ \\
Others & $4(10 \%)$ \\
\hline Requested LAs & \\
Lidocaine & $22(54 \%)$ \\
Ropivacaine & $4(10 \%)$ \\
Unknown (Lidocaine was tested) & $15(36 \%)$ \\
\hline
\end{tabular}


Table 5 Clinical Data of Six Patients with Test Positivity to LAs

\begin{tabular}{|l|l|l|l|l|l|l|l|}
\hline No. & Age & Gender & Procedure & Clinical Characteristics & Culprit Drug & Skin Tests & BAT \\
\hline I & 34 & F & Surgery & Hypotension, palpitations, shock, angioedema & Ropivacaine & Positive & Positive \\
2 & 25 & F & Dentistry & Hypotension, palpitation, dizziness, dyspnea, somnolence & Lidocaine & Positive & Negative \\
3 & 52 & F & Surgery & Hypotension, rash & Ropivacaine & Positive & Negative \\
4 & 75 & F & Surgery & Tachycardia, malaise, syncope & Lidocaine & Positive & Negative \\
5 & 25 & F & Dentistry & Dizziness, angioedema & Lidocaine & Positive & Positive \\
6 & 29 & F & Dentistry & Hypotension, rash & Lidocaine & Not done & Positive \\
\hline
\end{tabular}

symptoms. However, two patients showed no skin symptoms (Table 5).

According to the skin tests of 99 (91\%) patients in this study, five patients had allergic reactions. Of those patients, three cases had a positive IDT with lidocaine, while two cases with ropivacaine. According to the BAT results of $103(94 \%)$ patients in this study, three cases were positive. Furthermore, one patient had a positive BAT but skin tests were not done, the culprit drug was lidocaine (Table 5).

\section{Discussion}

A total of 109 patients who underwent tests with LAs in our anesthesia allergy clinic were included in the analysis, only six out of these patients presented true type allergy to LAs. The results showed that risk of true allergy to LAs is very low. A meta-analysis recently showed that true allergy to LAs are extremely rare, it assessed 23 case series involving 2,978 patients between 1950 and 2011, only 29 of these patients presented true allergy to LAs. ${ }^{16}$ Although our study was only a summary of the test results in the past decade, it has important potential implications for clinical practice. The DHR during anesthesia and operation, especially anaphylaxis, may be dangerous or even life-threatening to patients and result in lifelong sequelae. Suspected history of DHR to LAs may prompt patients and doctors to reject future LAs injections. If the culprit drug could be investigated and diagnosed promptly and correctly, severe adverse events may be avoided in the follow-up operation. In the current shortage of allergy testing resources in China, it is reasonable to target highrisk patients and those who may have serious complications in the case of allergic reactions during anesthesia procedures. Therefore, despite the low frequency of true allergy to LAs, patients with a suspected history of DHR to LAs should be tested with LAs.

The finding from the Danish Allergy Clinic suggest that adverse drug reactions after administering LAs are usually caused by other nonallergic mechanisms in most cases. ${ }^{1}$
These include overdose toxicity with paresthesia and dizziness, vasovagal response manifested as hypotension and syncope, incorrect drug administration, and the influence of adrenaline which is commonly co-administered to increase local duration of action and can lead to palpitations and tachycardia. ${ }^{17}$ Each adverse reaction could be confused with allergic reactions to LAs. However, none of these above reactions show skin symptoms. In other words, if adverse reactions to LAs present skin symptoms, such as rash or urticaria, combined with cardiovascular and/or respiratory symptoms, there is a higher risk of identifying allergic mechanisms. Therefore, doctors should immediately identify these systemic reaction symptoms, especially skin symptoms, correctly diagnose and manage patients, and start treatment with the intravenous administration of adrenaline. It is an important differential diagnosis for anesthesiologists to keep in mind and still a major problem in daily clinical practice.

Patients who are allergic to other drugs, especially general anesthesia drugs, are considered high risk groups for allergy to LAs. ${ }^{18}$ However, our data showed that skin tests and BAT results were negative for all 41 patients with a history of DHR to other drugs, the results excluded the IgE-mediated allergic reactions. Recently several studies suggested that only a previous history of HDR after exposure to LAs is considered to be at risk of similar or even more severe reactions. ${ }^{19,20}$ A larger cohort study may be needed in the future to determine this potential risk. Guidelines suggested that patients should not be tested for LAs, unless they had a previous history of LAs hypersensitivity. ${ }^{11,13}$ In our daily clinical practice, we noted that some patients who had a previous history of DHR to other drugs were referred to our clinic before a local anesthesia procedure. For high-risk patients or those who may have severe adverse events during anesthesia and operation (such as cardiovascular/cerebrovascular events), they underwent tests with LAs to ensure that requested LAs can be used safely in the future. 
The perioperative environment is very complex, and the patient's exposures include anesthetic drugs and other substances. In this case, LAs can present as a potential "hidden allergen", due to lubricating gels and sprays used for endoscopy often containing LAs, but it is rarely recorded on charts. ${ }^{21}$ Furthermore, it has been emphasized that the importance of identifying other simultaneous exposures, which is an important aspect of clinical management in these patients with suspected DHR to LAs. ${ }^{17}$ In fact, other drugs and substances commonly used in local anesthesia procedures, such as antibiotics, chlorhexidine, latex, or excipients, are more likely to cause allergic reactions. ${ }^{1,22,23}$ One of the limitations of our research is the lack of evaluation of these agents. Epidemiological studies to assess the incidence and causes of true allergy to LAs have been difficult to conduct, because the other suspected agents are usually unknown and rarely recorded on most medical charts, ${ }^{24}$ just as in our cases. The lack of these details seriously hinders the correct diagnosis, so it is essential for the close cooperation between allergists and anesthesiologists to conduct highly professional and detailed investigations of suspected allergic reactions.

At present, there are several methods for the diagnosis of drug allergy. However, making the diagnosis is still a complex and challenging issue. Generally, investigation protocols differ among studies and many rely on skin tests, which is considered the Gold Standard, but they cannot be regarded as an absolute diagnosis due to the high rate of false positives. ${ }^{25}$ And current commercial immunoassays for determining specific IgE levels can only be applied to a restricted number of drugs used during anesthesia. ${ }^{26}$ Moreover, LAs-specific-IgE assays are not validated, so it is meaningful to develop other reliable diagnostic methods. ${ }^{27}$ The Basophil activation test (BAT), flow cytometry-assisted technique to quantify the CD63 expression on basophils, may be a new and promising in vitro method for the diagnosis of allergic reactions. ${ }^{15,28}$ The BAT could be used as a supplement to skin tests, especially when skin tests are not available or presented equivocal results, a positive result in BAT may identify the culprit agent. ${ }^{28,29}$ According to the BAT results of 103 patients in this study, three cases were positive. The results suggest that BAT may be useful in the investigation and diagnosis of true allergy to LAs. However, the diagnostic sensitivity of BAT is still unclear, it remains in the experimental stage and has not become a widely used technique in daily clinical practice. ${ }^{27}$ It is reported that the specificity of BAT is very high (93-100\%), but the sensitivity varies in several studies $(63-89.7 \%)$ that evaluate BAT in the diagnosis of allergy to neuromuscular blocker (NMBAs). ${ }^{30-32}$ Furthermore, as far as we know, there are no studies to evaluate the use of BAT for routine diagnosis of LAs allergy in China. The investigation of the culprit drug for allergic reactions is still quite difficult, and additional studies need to be done in future to evaluate the sensitivity of BAT.

\section{Conclusions}

Over the past 10 years, many patients have been referred to our clinic for allergy tests to find safe LAs that could be used for upcoming surgery. This study showed that risk of true allergy to LAs may be very low. However, patients with a suspected history of DHR to LAs should be considered for allergy tests with LAs. It is necessary to highlight the importance of detailed investigation and complete allergy tests for correct diagnosis and treatment. Skin tests and BAT may be reliable methods for investigation and diagnosis of true allergy to LAs in clinical practice, and this testing procedures could contribute to identifying the extremely rare cases of LAs allergy.

\section{Ethical Approval}

The Ethics Committee of China-Japan Friendship Hospital consented to review the anonymous clinical data retrospectively and approved the study (2019-108-K76). Informed consent was not required for a retrospective study. This study was conducted in accordance with the Declaration of Helsinki. All patient data was guaranteed to be confidential, and the researchers were committed to protecting the privacy and personal identity information of patients.

\section{Funding}

There is no funding to report.

\section{Disclosure}

The authors report that they have no conflicts of interest in this work.

\section{References}

1. Kvisselgaard AD, Mosbech HF, Fransson S, Garvey LH. Risk of immediate-type allergy to local anesthetics is overestimated-results from 5 years of provocation testing in a danish allergy clinic. J Allergy Clin Immunol Pract. 2018;6(4):1217-1223. 
2. Janas-Naze A, Osica P. The incidence of lidocaine allergy in dentists: an evaluation of 100 general dental practitioners. Int J Occup Med Environ Health. 2019;32(3):333-339.

3. Fellinger C, Wantke F, Hemmer W, et al. The rare case of a probably true ige-mediated allergy to local anaesthetics. Case Rep Med. 2013;2013:201586. doi:10.1155/2013/201586

4. Venemalm L, Degerbeck F, Smith W. IgE-mediated reaction to mepivacaine. J Allergy Clin Immunol. 2008;121(4):1058-1059. doi:10.1016/j.jaci.2007.12.1154

5. Haugen RN, Brown CW. Case reports: type I hypersensitivity to lidocaine. J Drugs Dermatol. 2007;6(12):1222-1223.

6. Solensky R, Khan DA. Drug allergy: an updated practice parameter. Ann Allergy Asthma Immunol. 2010;105(4):259-273.e78.

7. Harboe T, Guttormsen AB, Aarebrot S, et al. Suspected allergy to local anaesthetics: follow-up in 135 cases. Acta Anaesthesiol Scand. 2010;54(5):536-542. doi:10.1111/j.1399-6576.2009.02193.x

8. Batinac T, Sotošek Tokmadžić V, Peharda V, et al. Adverse reactions and alleged allergy to local anesthetics: analysis of 331 patients. $J$ Dermatol. 2013;40(7):522-527. doi:10.1111/1346-8138.12168

9. Kvisselgaard AD, Krøigaard M, Mosbech HF, et al. No cases of perioperative allergy to local anaesthetics in the Danish anaesthesia allergy centre. Acta Anaesthesiol Scand. 2017;61(2):149-155. doi:10.1111/aas.12833

10. Brockow K, Romano A, Blanca M, Ring J, Pichler W, Demoly P. General considerations for skin test procedures in the diagnosis of drug hypersensitivity. Allergy. 2002;57(1):45-51.

11. Brockow K, Garvey LH, Aberer W, et al. Skin test concentrations for systemically administered drugs - an ENDA/EAACI Drug Allergy Interest Group position paper. Allergy (Copenhagen). 2013;68 (6):702-712.

12. YIlmaz I, Ozdemir SK, Aydin O, et al. Local anesthetics allergy: who should be tested? Eur Ann Allergy Clin Immunol. 2017;50(2):66-71. doi:10.23822/EurAnnACI.1764-1489.38

13. Demoly $\mathrm{P}$, Adkinson NF, Brockow K, et al. International consensus on drug allergy. Allergy. 2014;69(4):420-437. doi:10.1111/all.12350

14. Furci F, Martina S, Faccioni P, et al. Adverse reaction to local anaesthetics: is it always allergy? Oral Dis. 2020;26(6):1340-1342. doi:10.1111/odi.13310

15. Xin X, Zou Y, Xing L, et al. Investigation of drugs responsible for perioperative anaphylactic reactions using cellular allergen stimulation test. Chin Med J. 2014;127(21):3738-3743.

16. Bhole MV, Manson AL, Seneviratne SL, et al. IgE-mediated allergy to local anaesthetics: separating fact from perception: a UK perspective. $\mathrm{Br}$ J Anaesth. 2012;108(6):903-911. doi:10.1093/bja/aes162

17. Saff RR. Immediate local anesthetic reactions: too quick to point the finger? J Allergy Clin Immunol Pract. 2018;6(4):1224-1225.

18. Wöhrl S, Vigl K, Stingl G. Patients with drug reactions - is it worth testing? Allergy. 2006;61(8):928-934. doi:10.1111/j.1398-9995.2006. 01148.x
19. Berkun Y, Ben-Zvi A, Levy Y, et al. Evaluation of adverse reactions to local anesthetics: experience with 236 patients. Ann Allergy Asthma Immunol. 2003;91(4):342-345. doi:10.1016/S1081-1206(10) 61680-8

20. Grzanka A, Wasilewska I, Śliwczyńska M, Misiołek H. Hypersensitivity lo local anesthetics. Anaesthesiol Intensive Ther. 2016;48(2):128-134. doi:10.5603/AIT.a2016.0017

21. Kvisselgaard AD, Melchiors BB, Krøigaard M, et al. Lidocaine as a rare and hidden allergen in the perioperative setting. A A Practice. 2019;12(12):430-432. doi:10.1213/XAA.0000000000000955

22. Thyssen JP, Menné T, Elberling J, et al. Hypersensitivity to local anaesthetics - update and proposal of evaluation algorithm. Contact Dermatitis. 2008;59(2):69-78. doi:10.1111/j.1600-0536.2008.01366.x

23. Parkes AW, Harper N, Herwadkar A, et al. Anaphylaxis to the chlorhexidine component of Instillagel $^{\circledR}$ : a case series. $\mathrm{Br}$ J Anaesth. 2009;102(1):65-68. doi:10.1093/bja/aen324

24. Garvey LH, Old N. Hidden causes of perioperative hypersensitivity. Curr Pharm Des. 2016;22(45):6814-6824. doi:10.2174/13816128 22666161004125143

25. Mertes PM, Malinowsky JM, Jouffroy L, et al. Reducing the risk of anaphylaxis during anesthesia: 2011 updated guidelines for clinical practice. J Investig Allergol Clin Immunol. 2011;21(6):442.

26. Mayorga C, Ebo DG, Lang DM, et al. Controversies in drug allergy: in vitro testing. J Allergy Clin Immunol. 2019;143(1):56-65. doi:10. 1016/j.jaci.2018.09.022

27. Mayorga C, Celik G, Rouzaire P, et al. In vitro tests for drug hypersensitivity reactions: an ENDA/EAACI Drug Allergy Interest Group position paper. Allergy. 2016;71(8):1103-1134. doi:10.1111/ all.12886

28. Hoffmann HJ, Santos AF, Mayorga C, et al. The clinical utility of basophil activation testing in diagnosis and monitoring of allergic disease. Allergy. 2015;70(11):1393-1405. doi:10.1111/all.12698

29. Eberlein B, Wigand S, Lewald H, et al. Utility of basophil activation testing to assess perioperative anaphylactic reactions in real-world practice. Immun Inflamm Dis. 2017;5(4):416-420. doi:10.1002/ iid3.175

30. Monneret G, Benoit Y, Debard AL, et al. Monitoring of basophil activation using CD63 and CCR3 in allergy to muscle relaxant drugs. Clin Immunol. 2002;102(2):192-199. doi:10.1006/clim.2001.5156

31. Ebo DG, Bridts CH, Hagendorens MM, et al. Flow-assisted diagnostic management of anaphylaxis from rocuronium bromide. Allergy. 2006;61(8):935-939. doi:10.1111/j.1398-9995.2006.01094.x

32. Sudheer PS, Hall JE, Read GF, et al. Flow cytometric investigation of peri-anaesthetic anaphylaxis using CD63 and CD203c. Anaesthesia. 2005;60(3):251-256. doi:10.1111/j.1365-2044.2004.04086.x
Therapeutics and Clinical Risk Management

\section{Publish your work in this journal}

Therapeutics and Clinical Risk Management is an international, peerreviewed journal of clinical therapeutics and risk management, focusing on concise rapid reporting of clinical studies in all therapeutic areas, outcomes, safety, and programs for the effective, safe, and sustained use of medicines. This journal is indexed on PubMed Central, CAS,
EMBase, Scopus and the Elsevier Bibliographic databases. The manuscript management system is completely online and includes a very quick and fair peer-review system, which is all easy to use. Visit http://www.dovepress.com/testimonials.php to read real quotes from published authors. 